\title{
Simulation of Drive System with Self-Tuning Fuzzy PID Control Application to PMSM
}

\author{
Hui Hang and Bingyi Zhang \\ School of Electrical Engineering, Shenyang University of Technology, Shenyang 110870, P.R. China
}

\begin{abstract}
A permanent magnet synchronous motor (PMSM) drive control system for drive machine tools includes nonlinearities, uncertainties and external perturbations that should be considered in the design of control laws. A quantification factor self-tuning fuzzy PID controller for drive system is suggested by analysis the deficiency and characteristics of conventional PID controller and the limitations of conventional fuzzy PID controller. The simulation results show that the controller can map the error to the fuzzy domain in the whole speed reference range, and slow the system down when the error is small. There is almost no overshoot in both haste and slow process. The proposed controller is superior to the conventional fuzzy PID controller in dynamic stability performance and speed tracking power, and the quantification factor self-tuning fuzzy PID controller has strong robustness to external disturbance.
\end{abstract}

Keywords-component; PMSM; quantification factor; selftuning; fuzzy PID; robustness

\section{INTRODUCTION}

In recent years, the permanent magnet synchronous motors (PMSM) are more and more used in high performance industrial drive systems ranging from small servo drives to high machine tool drives. The main reason of the increasing interest is their high efficiency and the higher power factor. However, the PMSM presents a coupled nonlinear multivariable control structure which calls for complex nonlinear design in order to get good dynamic performance[1][2]. Moreover, the performances must be insensitive to the drive and load parameter variation. Vector control is typically used in these drives to improve the dynamic response and give the performance characteristics similar to that of a DC machine which are desirable in certain applications. The high performance drive system necessitate quickly response and robustness to parameters variation. However the conventional PID controllers of fixed parameters are not able to meet the needs of high accuracy in computer numerical control system and PID controller cannot solve the disturbance bring by timevarying system. To overcome this problem, modern regulators are used, like fuzzy logic controller. Fuzzy control with a small dependence on the controlled object can adjust the control rules according to the control results and have the advantages of robustness. Based on the characteristics of PMSM, combining with the advantage of PID control and fuzzy controller a quantification factor self-tuning fuzzy PID controller was proposed in this paper.

\section{MATHEMATICAL MODEL OF PMSM}

The mathematical model of three- phase PMSM is presented in the following equations. Taking into account the complexity of the model, make the following assumptions [3]:

(1)Neglecting stator and rotor core reluctance, eddy current and hysteresis losses;

(2)The conductivity of permanent magnet material is zero, and the internal magnetic permeability of it is the same as air.

(3)The excitation field generated by the magnetic field and reaction magnetic field in the armature in the gap is sinusoidal distribution;

(4)The phase winding induced voltage waveform is sinusoidal at steady state.

Now, three-phase winding voltage and torque equation can be expressed as:

$$
\begin{aligned}
& u_{\mathrm{A}}=R_{\mathrm{s}} i_{\mathrm{A}}+\frac{d \psi_{\mathrm{A}}}{d t} \\
& u_{\mathrm{B}}=R_{\mathrm{s}} i_{\mathrm{B}}+\frac{d \psi_{\mathrm{B}}}{d t} \\
& u_{\mathrm{C}}=R_{\mathrm{s}} i_{\mathrm{C}}+\frac{d \psi_{\mathrm{C}}}{d t} \\
& T_{\mathrm{e}}=p_{0} \psi_{\mathrm{f}} \times i_{\mathrm{s}}
\end{aligned}
$$

Mathematical model in three-phase PMSM is a nonlinear, high order, strong coupling model, the control is extremely complex. The mathematical model under the $\mathrm{d}-\mathrm{q}$ axis is obtained by coordinate transformation. The mathematical model is as follows:

$$
\begin{gathered}
u_{\mathrm{d}}=R_{\mathrm{s}} i_{\mathrm{d}}+p L_{\mathrm{d}} i_{\mathrm{d}}-\omega_{\mathrm{r}} L_{\mathrm{q}} i_{\mathrm{q}} \\
u_{\mathrm{q}}=R_{\mathrm{s}} i_{\mathrm{q}}+p L_{\mathrm{q}} i_{\mathrm{q}}+\omega_{\mathrm{r}} L_{\mathrm{d}} i_{\mathrm{d}}+\omega_{\mathrm{r}} \psi_{\mathrm{f}}
\end{gathered}
$$




$$
\begin{gathered}
T_{\mathrm{e}}=\frac{3}{2} p_{0} \psi_{\mathrm{f}} i_{\mathrm{q}} \\
T_{\mathrm{e}}-T_{\mathrm{L}}=J \frac{d \omega_{\mathrm{m}}}{d t}+B \omega_{\mathrm{m}}
\end{gathered}
$$

where $u_{\mathrm{d}}, u_{\mathrm{q}}$ corresponds to phase voltage in direct axis and cross-axis separately (V); $L_{\mathrm{d}}, L_{\mathrm{q}}$ corresponds to phase inductance in direct axis and cross-axis separately $(\mathrm{H}) ; \Psi_{\mathrm{f}}$ corresponds to rotor flux $(\mathrm{Wb}) ; \omega_{\mathrm{m}}$ corresponds to rotor mechanical speed $(\mathrm{rad} / \mathrm{s}), \omega_{\mathrm{r}}$ corresponds to rotor electrical speed, where $\omega_{\mathrm{r}}=p_{0 \times} \omega_{\mathrm{m}} ; p_{0}$ corresponds to number of pole pairs, $J$ is rotor inertia $\left(\mathrm{kg} \bullet \mathrm{m}^{2}\right), B$ is the friction coefficient $(\mathrm{N} \bullet \mathrm{m} \bullet \mathrm{s}), T_{\mathrm{L}}, T_{\mathrm{e}}$ represents load torque and electromagetic torque separately $(\mathrm{N} \bullet \mathrm{m})$.

A surface-mounted PMSM is used in this paper, the direct axis inductance and cross axis inductance is nearly the same. Mathematical model of surface-mounted PMSM can be obtained by make $L_{\mathrm{d}}=L_{\mathrm{q}}=L_{\mathrm{s}}$.

\section{VECTOR CONTROL SYSTEM OF PMSM}

Vector control principle was first proposed by the German engineer Blaschke, and extended to permanent magnet synchronous motor control system. Vector control for PMSM is easier and the performance is more favorable than the induction motor because the rotor flux is constant and little affected by temperature. The basic idea of vector control is to make the torque current and exciting current forming in the space of 90 electrical angle degrees in $\mathrm{d}-\mathrm{q}$ coordinate system through coordinate transformation to achieve decoupling control of stator current. Then AC motor is equivalent to its excitation DC motor. The system control block diagram is shown in Figure I.

\section{SYSTEM DESIGN}

In this paper, the conventional fuzzy PID controller and quantification factor self-tuning fuzzy PID controller were designed, in which the parameters of conventional PID controller were designed by the engineering method, and using trial and error method to determine the final control parameters. The parameters of fuzzy PID controller are based on the designed conventional PID controller.

\section{A. Conventional PID Control}

PID controller is linear combinations of proportional, integral and differential.

Even if the parameters are tuned to good values, it cannot meet the requirements of the servo system, because there are many uncertain factors in the permanent magnet synchronous motor feeding system, such as sudden load disturbances and others [4][5].

\section{B. Quantification Factor Self-Tuning Fuzzy PID Control} Once the traditional fuzzy controller is designed, its fuzzy and de-fuzzy quantization factors are fixed, and fixed factor controller cannot change the continuous input to fuzzy domain throughout the speed range properly, then fuzzy controller will make inaccurate decision, which will reduce the adaptive ability of the system. The purpose of this paper is to improve the adaptive ability of fuzzy PID controller.

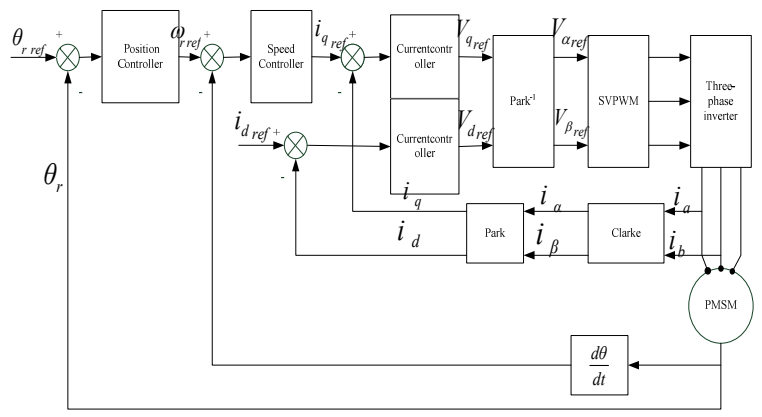

FIGURE I. VECTOR CONTROL SYSTEM BLOCK DIAGRAM

In this paper, the speed error $e$ and the change rate of error $e c$ are inputs of fuzzy PID controller and the $d K_{\mathrm{p}}, d K_{I}, d K_{d}$ are outputs. The fuzzy controller will adjust the parameters of PID controller on line according to the designed inference rules. The control block is shown in figure II.

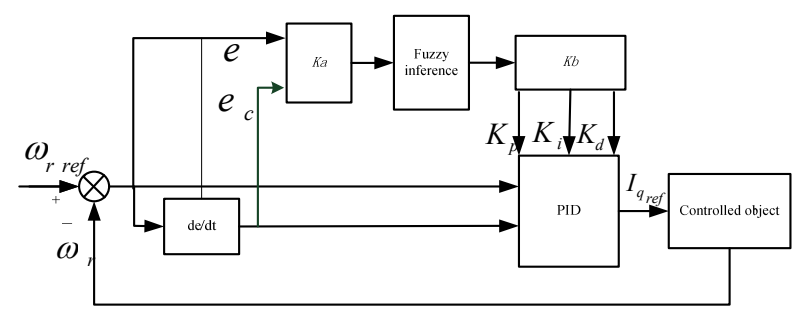

FIGURE II. BLOCK OF FUZZY PID CONTROLLER

\section{1) Derivation of Fuzzy Control Rules}

According to the previous experience and combining with the principle of quantification factor self-tuning, we can obtain the following general principles:

When the absolute value of error $e$ is large, we should increase $K_{p}$, and decreases $K_{I}$ and $K_{d}$ to improve speed tracking, reduce integral saturation effect and overshoot and prevent the system slowing down in advance.

If the error is medium, then $K_{I}$ and $K_{d}$ should be increased appropriately to strengthen predictive effect. If error is small (error $=8 \% *$ reference speed), and the error change rate is big. Then we should appropriately increase $K_{d}$, reduce $K_{p}$, and $K_{\mathrm{I}}$ to make the system slow down, de-saturation the current regulator and reduce the overshoot. When error closes to zero, we must increase $K_{p}, K_{I}$ and reduce the $K_{d}$ in order to improve steadystate accuracy and noise-immunity.

Quantification factor $K_{a}$ of conventional fuzzy PID controller is fixed, so it is not able to map the error to proper value in fuzzy domain, and it will cause that the fuzzy PID is not adaptive to varying command.

For example, $\omega_{\mathrm{e} \text { ref }}=1000$, quantization factor $K_{a}=0.6 \%$, 
then error value 1000 corresponding to 6 , when the error value is 100 then it corresponds to $0.6, \mathrm{ZO}$ (zero) belongs to $[-1,1]$ in the general control rules, then the system will think that the error has been close to zero, and it will increase $K_{p}$ and $K_{I}$ to reduce the steady-state error, which would cause the system to overshoot.

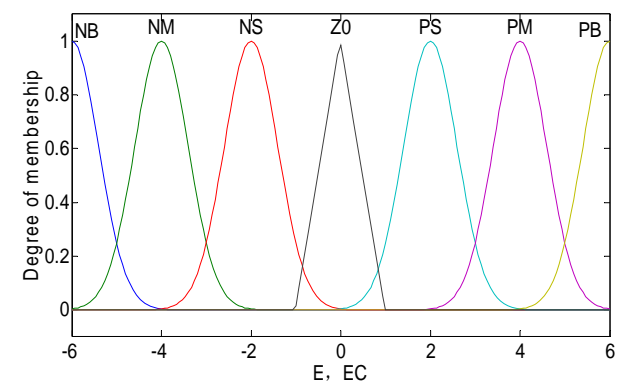

(A) MF OF INPUTS E,EC

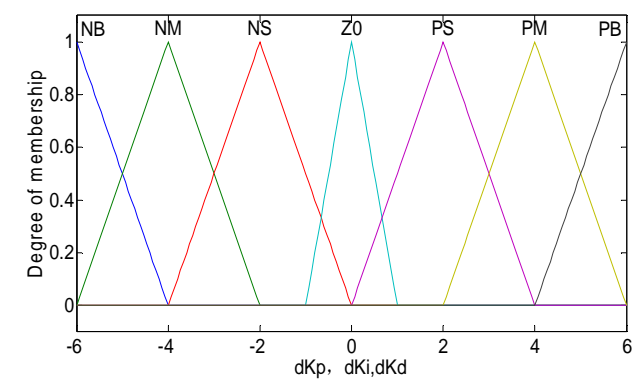

(B) MF OF OUTPUTS $d K p, d K_{I}, d K_{d}$

FIGURE III. MEMBERSHIP FUNCTIONS

To solve the insufficient adaptive ability of fuzzy control, a simple principle of factor self-tuning is proposed for first time in this paper. According to this rule, the fuzzy controller can accurately map the error to the fuzzy domain; and make the right judgments when the error is small to make the system slow down to achieve little or no overshoot purposes. The system will slow down when the error is $8 \%$ of the reference, so there is no effect on the system quickness and the speed is highly increased when the error is big and medium. in this paper when error is $8 \%$ of reference, the controller will judge that the error is small, and it should increase $K_{\mathrm{d}}$ and decrease $K_{p}$ and $K_{I}$ to slow down the speed to reduce overshoot. After that, the error will be close to zero and the controller will increase $K_{p}, K_{I}$, and reduce $K_{d}$ to improve steady-state accuracy and robustness.

Factor $K_{a}$ is designed in accordance with the following equation: $K a \times($ reference $\times 8 / 100)=2 \quad$. Therefore, the quantification factor of the fuzzy controller is self-tuning based on reference on line, the system will slow down to reduce the overshoot when the error is $8 \%$ of the reference. This speed controller can be ported to the position loop, because the position loop requires no overshoot. Specific fuzzy control rules are shown in Table I-III.

\section{2) Defuzzification}

The inferred results are also fuzzy variables and they should be converted to the crisp set. In this paper, the center of gravity defuzzification is used. The output function is given in (9).

Take $d K_{p}$ for example:

$$
d k_{p}=\frac{\sum_{n=1}^{p}\left[\Delta k_{p \mathrm{n}} \mu\left(\Delta k_{p \mathrm{n}}\right)\right]}{\sum_{i=1}^{p} \mu \mathrm{i}}
$$

where $d K_{p}$ is clarity output, $K_{p}$ is the output variables, $\mu$ is fuzzy membership function and $p$ is the number of single-point set [6].

TABLE I. CONTROL RULES FOR DKP

\begin{tabular}{|c|ccccccc|}
\hline$d K_{P}$ & & & & $E C$ & & & \\
\hline E & NB & NM & NS & ZO & PS & PM & PB \\
\hline NB & PB & PB & PM & PM & PS & ZO & ZO \\
\hline NM & PB & PB & PM & PS & PS & ZO & NS \\
\hline NS & ZO & PM & PM & PS & ZO & ZO & NS \\
\hline ZO & NS & PS & PS & PB & NS & NM & PM \\
\hline PS & ZO & PS & ZO & NS & NS & NM & NM \\
\hline PM & PS & ZO & NS & NM & PM & PM & NB \\
\hline PB & ZO & PS & PB & PB & NB & NB & NB \\
\hline
\end{tabular}

TABLE II. CONTROL RULES FOR DKI

\begin{tabular}{|c|ccccccc|}
\hline$d K_{I}$ & \multicolumn{7}{|c|}{$E C$} \\
\hline E & NB & NM & NS & ZO & PS & PM & PB \\
\hline NB & NB & NB & NM & NM & NS & ZO & ZO \\
\hline NM & NB & NB & NM & NS & NS & ZO & ZO \\
\hline NS & NB & NM & NM & PS & ZO & PS & PS \\
\hline ZO & NM & ZO & ZO & PB & PS & PM & PS \\
\hline PS & NM & ZO & ZO & ZO & PS & PS & ZO \\
\hline PM & ZO & ZO & PS & PS & PB & PB & PB \\
\hline PB & ZO & ZO & PS & PM & PB & PB & PB \\
\hline
\end{tabular}

TABLE III. CONTROL RULES FOR DKD

\begin{tabular}{|c|ccccccc|}
\hline$d K_{d}$ & \multicolumn{7}{|c|}{$E C$} \\
\hline $\mathrm{E}$ & NB & NM & NS & ZO & PS & PM & PB \\
\hline NB & PS & NS & NB & NB & NB & NM & PS \\
\hline NM & PS & NS & NB & NM & PS & PS & PS \\
\hline NS & ZO & NS & NS & NM & PS & PB & PB \\
\hline ZO & PM & PS & PM & ZO & PS & PM & PB \\
\hline PS & PB & PB & PM & PB & ZO & PB & PB \\
\hline PM & ZO & ZO & ZO & ZO & ZO & ZO & ZO \\
\hline PB & ZO & ZO & ZO & ZO & ZO & ZO & ZO \\
\hline
\end{tabular}

\section{Simulations}

In this paper, vector control system of PMSM include current loop, speed loop and coordinate transformation block, electrical machine block and measuring blocks. a PI speed 
controller and a quantification factor self-tuning fuzzy PID speed controller respectively and a current hysteresis comparator were designed.

By the limited space, electrical machine and measuring block, coordinate transformation block cannot be introduced.

Simulation parameters of PMSM: $R_{\mathrm{S}}=4.673330 \mathrm{hm}, L_{\mathrm{d}}=$ $27.627 \mathrm{mH}, L_{\mathrm{q}}=25.993 \mathrm{mH}, J=2.8642 \mathrm{Kg} . \mathrm{m}^{2}$, rotor flux $\Psi_{\mathrm{f}}=$ $0.275 \mathrm{~Wb}$, magnet pole pairs $p_{0}=22$, friction coefficient $B=0.01$ $\mathrm{N} \cdot \mathrm{s} \cdot \mathrm{m}$.

\section{A. Current Hysteresis Comparator}

The current loop is the inner loop of a system, its fundamental role is to track the reference, and keep current under the limit value, its requirement is rapid response. In this paper DC bus voltage is $350 \mathrm{~V}$, current limit range: [-30A, 30A] Hysteresis current comparator block is shown in Figure IV.

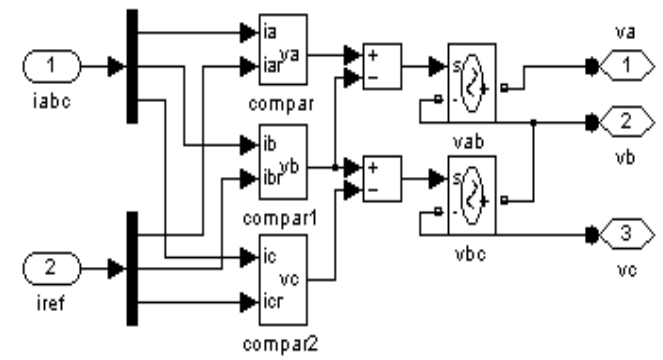

FIGURE IV. CURRENT HYSTERESIS COMPARATOR

\section{B. Quantification Factor Self-Tuning Fuzzy PID Controller}

Through trial and error we can obtain the parameters of PID controller, the principle of tuning is to adjust the proportional coefficient to make the system respond fast and overshoot little, then increase the integral coefficient until the error is eliminate quickly. The final choice: $K_{p}=10, K_{I}=2, K_{d}=0$. The principle of quantification factor self-tuning have been described in the fuzzy inference rules, we will not repeat them here. Quantification factor of error change rate is decide by observing the value of it, and the error change rate is between $10^{5}$ and $10^{6}$, so its quantification factor value is obtained as $10^{-5}$. Quantification factors of defuzzification were determined according to the initial value of PID parameters. $K_{I}$ can be reduce to zero, $K_{p}$ can be 2 times of the initial value and $K_{d}$ is about $10^{-5}$ is the aim. The final choice: $K_{d K p}=3.5, K_{d K I}=0.35$, $K_{d K d}=10^{-5}$. The control block is shown in figure V.

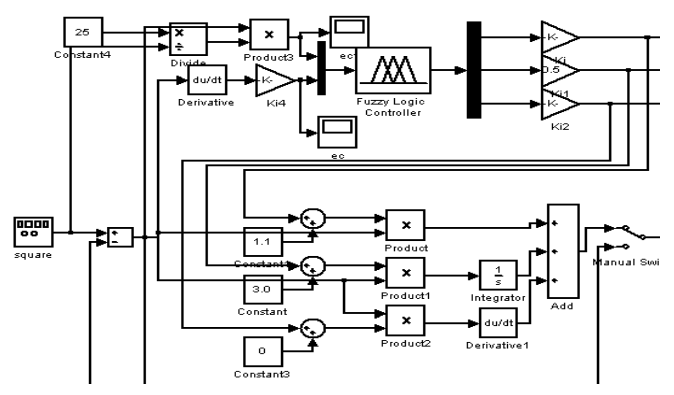

FIGURE V. QUANTIFICATION FACTOR SELF-TUNING FUZZY PID CONTROLLER

\section{RESUlTS ANALYSIS AND CONCLUSIONS}

Conventional fuzzy PID controller and quantification factor self-tuning fuzzy PID controller of PMSM simulation results are shown in Figure VI - VII. Figure VI shows the speed tracking comparison, the red line represent the quantification factor self-tuning fuzzy PID control (QFSFPIDC) and the blue line correspond to the conventional fuzzy PID control (FPIDC).

The two controllers have the same acceleration because of current limiting effect, each rise time is nearly the same, however, the adjustment time of QFSFPIDC is much shorter than the conventional fuzzy PID controller and overshoot of the proposed controller is also much smaller than fuzzy PID controller.

Figure VI shows speed tracking performance of both systems at 100rad / s, 50rad / s and 20rad/s. It can be seen from the figure, compared with normal fuzzy PID, QFSFPIDC has much smaller overshoot in the whole reference range. However, the performance of conventional fuzzy PID is poor in lower speed, the performance of QFSFPIDC is much better than FPIDC in overshoot and adjustment time.

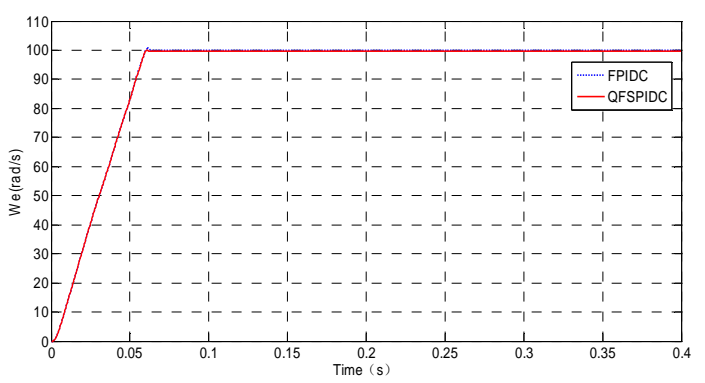

(a) $100 \mathrm{rad} / \mathrm{s}$

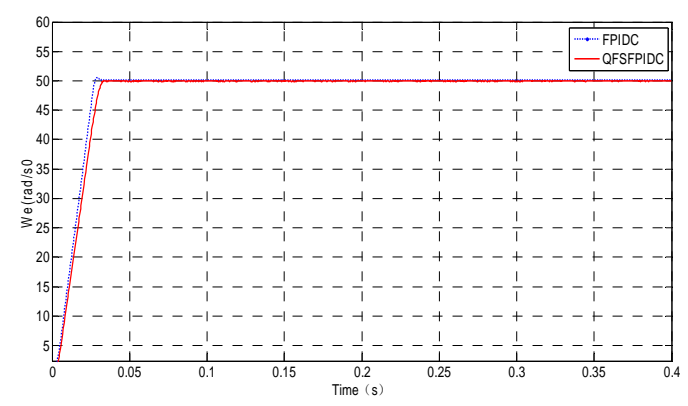

(b) $50 \mathrm{rad} / \mathrm{s}$

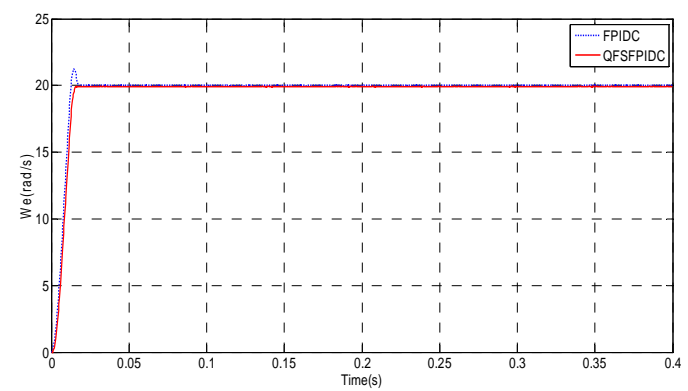

(c) $20 \mathrm{rad} / \mathrm{s}$

FIGURE VI. COMPARISION OF SPEED TRACKING 
Disturbance of load torque is shown in figure VII; when the sudden load torque of $30 \mathrm{~N} \bullet \mathrm{m}$ is add to the system at $0.08 \mathrm{~s}$, speed of the PI controller dropped $1.36 \%$, and conventional fuzzy PID as $0.4 \%$, while the QFSFPIDC is only $0.2 \%$. Torque fluctuation of fuzzy PID is up to $15 \mathrm{~N} \cdot \mathrm{m}$, while the QFSFPIDC is only $5 \mathrm{~N} \bullet \mathrm{m}$, the specific comparison is shown in Figure VII.

PMSM drive system has strong nonlinearity and perturbation of system parameters, load disturbances and other uncertainties making the fuzzy PID controller difficult to achieve high performance of speed.

Based on the characteristics of PMSM, combining with the advantage of PID control and fuzzy controller a quantification factor self-tuning fuzzy PID controller was designed. Simulation results confirmed that performance of the proposed system is robust, stable, and insensitive to parameters and operating condition variations.

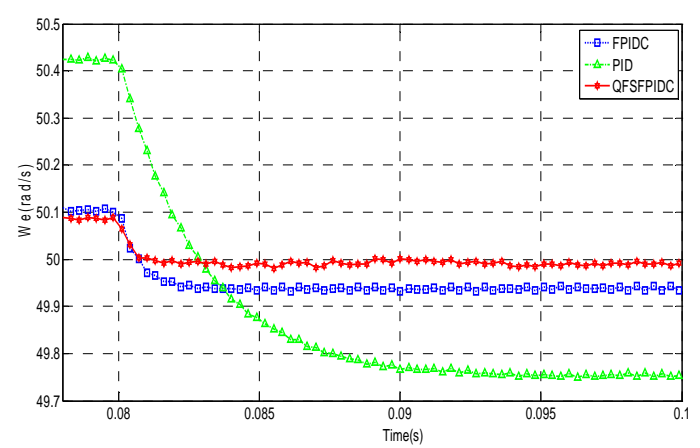

FIGURE VII. INFLUENCE OF LOAD TORQUE

What is more, the proposed controller is superior to the conventional fuzzy PID controller in immunity of operating condition variation.

\section{REFERENCES}

[1] P.Pillay,R.Krishnan, "Modulation, simulation and analysis of Permanent magnet motor drive,” IEEE Trans.on Ind. Appl.vol.25, no.2,pp. 265-273, Apr.1989.

[2] P. Guillemin, "Fuzzy logic applied to motor control," IEEE Trans. on Ind. Appl. vol.32, no.1,pp. 51-56, Feb.1996.

[3] Chengyuan WANG, Jiakuan XIA, Yibiao SUN, Modern Control Technique of Electric Machine, Beijing: China Machine Press, 2008.

[4] Boshi CHEN, Automatic control of electric drive system motion control system, Beijing: China Machine Press, 2003.

[5] Huacai LU, Yuetong XU, Weimin YAN , "Fuzzy PID Controller Design for a Permanent Magnet Linear Synchronous Motor Feeding System,” Trans. of china electrotechnical society, vol.22, no.4, pp.59-63, 2007.

[6] Houmei CUI, Xijin GUO, Kelin LIU, "Fuzzy-Adaptive PID Control in the Speed Control System,” Techniques of Automation \& Applications, vol.28, no.9, pp.101-104, 2009. 